\title{
Context in verbal short-term memory
}

\author{
BRADLEY R. POSTLE \\ University of Wisconsin, Madison, Wisconsin
}

\begin{abstract}
We tested the hypothesis that stimulus-related contextual information that is incidental to task demands - an episodic code - is automatically, obligatorily encoded and stored as a part of short-term memory (STM) representations. Four experiments employed a running span task to investigate the effects of manipulating two types of contextual information: stimulus grouping and color. Three experiments established that grouping context effects are sensitive neither to volitional control nor to task difficulty and that they generalize across testing procedures (yes/no recognition and immediate serial recall). A fourth experiment demonstrated an effect of manipulating the congruity of the color of stimuli between study and test. These demonstrations of the robustness and generality of context effects in STM are consistent with the predictions of the episodic coding model of STM.
\end{abstract}

Previous research with a running span (or "updating") working memory task has revealed a group integrity effect (Postle, Berger, Goldstein, Curtis, \& D’Esposito, 2001). Participants viewed serial presentation of items in groups of one, two, or three, and were instructed to maintain in memory, for subsequent recognition, the four most recently presented items. Performance was sensitive to whether items that had been presented together at encoding were also dropped together from the memory span, or whether this encoding-defined group was broken up when some of its items had to be dropped from the memory set while others were maintained. This group integrity effect was notable because the factor of group integrity was not described prior to testing, and the group-related context associated with an item was unrelated to task demands (i.e., attention to grouping context could not be an effective strategy). For this reason, we hypothesized that the group integrity effect was attributable to information about group context that was encoded automatically as a feature of stimulus representations in our task.

The goal of the present research was to assess the theoretical proposition that arose from this previous work: Episodic codes are fundamental components of representations in short-term memory (STM). ${ }^{1}$ To translate this proposition into an empirically testable hypothesis, we hypothesized that information about context is automatically and obligatorily encoded and stored along with each STM representation. This work fits into the tradition of research on the effects on memory of what Wickens (1987) referred to as "context alpha," in which "the context or environ-

Supported by a William F. Vilas Young Investigator Award (University of Wisconsin, Madison). B.R.P. thanks Yui-Chung Chan, Olufunsho Faseyitan, Nicki Fricano, Jong-Hye Han, Yee-Yan Hui, Bridget Lam, and Christian Stilp for assistance with programming, data collection, and scoring, and Michael C. Anderson, Arthur Glenberg, and Richard N. A. Henson for helpful discussions of this work. Correspondence should be addressed to B. R. Postle, Department of Psychology, University of Wisconsin, Madison, WI 53706-1696(e-mail: postle@wisc.edu). mental surround is essentially irrelevant to the central task, whose demand characteristics remain the same regardless of the context" (pp. 135, 138-139), as contrasted with what he referred to as "context beta," which is "needed to clarify the meaning of a particular word or group of words, or some action; otherwise the event, verbal or other, is ambiguous" (Wickens, 1987, p. 135). (Baddeley, 1982, has termed these two concepts "independent" and "interactive" context, respectively, and Gorfein, 1987, has called the latter "semantic" context). Also outside the scope of the episodic coding model is Gorfein's "environmental" context, because, in addition to context alpha, it also incorporates something akin to behavioral or task set. Although the effects of context manipulations on long-term memory performance have been studied extensively (for a review, see Smith \& Vela, 2001), less is known about such effects in STM. Thus, confirmation of our hypothesis might provide novel evidence about the complexity of encoding operations and stimulus representation in STM.

To test the hypothesis that information about context is automatically and obligatorily encoded and stored along with each STM representation, we performed four experiments. Experiment 1 tested whether the effects of context in STM can be suppressed with appropriate training. Experiment 2 tested whether the effects of context in STM are sensitive to task difficulty (by accelerating the pace of the task). Experiment 3 tested whether the effects of context in STM generalize across testing formats (by probing memory with immediate serial recall, as contrasted with the yes/no recognition procedure employed in Experiments 1 and 2). Finally, Experiment 4 tested whether the effects of context in STM generalize across domains of information (by varying the color of items, rather than group integrity).

\section{GENERAL METHOD}

\section{Participants}

All participants were recruited from the University of Wisconsin, Madison, community and were between 18 and 35 years of age. In- 
formed consent was acquired from each participant prior to testing. Participation was compensated with course credit or with money. No one participated in more than one experiment.

\section{The Updating Task}

All experiments reported here employed variants of the updating task. Task analysis indicates that this task requires the coordination of at least five discrete mental operations: (1) adding items to STM (i.e., encoding); (2) discarding items from STM; (3) repositioning items in STM; (4) storing items in STM; and (5) rehearsing items in STM. It is the discarding and repositioning operations that make this an updating task and that may require executive control processes not required within individual trials of "simple" tests of STM span or duration, such as digit span and delayed recognition tasks, respectively (Cowan, 2001; D’Esposito \& Postle, 1999, 2000). For this reason, many of those who have worked with this task have classified it as a working memory task (Kiss, Pisio, Francois, \& Schopflocher, 1998; Morris \& Jones, 1990; Postle et al., 2001; Salmon et al., 1996). The mechanistic details of the discarding and repositioning operations are not known. Discarding a representation from STM, for example, might simply be accomplished by shifting attention away from that representation (e.g., Cowan, 2001; McElree, 2001). Alternatively, it might require an active suppression or inhibition mechanism (e.g., Hasher \& Zacks, 1988; Jonides, Smith, Marshuetz, Koeppe, \& Reuter-Lorenz, 1998).

\section{Procedure}

Stimuli were drawn randomly for each trial from the set of 21 consonant letters. Memoranda were presented as lowercase letters for a duration of $500 \mathrm{msec}$ on the screen of a desktop computer (interstimulus interval [ISI] varied across experiments), and no items repeated as memoranda on a trial. One, two, or three lowercase letters were presented per presentation epoch. Letters in groups of two or three were presented simultaneously, in a horizontal row. Sequence of presentation epochs (by size) was varied pseudorandomly and was constrained by the factor of group integrity (see below). Stimuli were displayed as white letters on a black background. A total of 4,8 , or 12 stimuli were presented in each trial, followed by a probe stimulus that appeared as a capital letter bounded by asterisks. There were two possible trial durations per stimulus length: 4item 2-presentation epoch trials lasted $8 \mathrm{sec}$ (in 3.5-sec ISI experiments) from beginning to probe onset, 4-item 3-presentation epoch trials lasted $12 \mathrm{sec}$, and so on for 8-item 4- and 5-presentation epoch trials and for 12-item 6- and 7-presentation epoch trials. Participants were instructed to maintain a memory of the four most recently presented items and to press the "yes" (right index finger) or "no" (left index finger) button on a response device connected to the computer in order to indicate whether or not the probe matched an item in the memory set. They were also instructed to respond as quickly and accurately as possible, but not to sacrifice accuracy for the sake of speed. Valid and invalid probes were equiprobable. Invalid probes never matched an item that had been presented (with a lag greater than 4 items) on the same trial.

Because only trials presenting more than 4 items required the discarding and repositioning operations that are central to updating, the effect of trial length on accuracy indexes an updating effect. Previous research has established that performance on the updating task is reliably lower on trials presenting more items than the memory span as opposed to trials presenting only the number of items in the memory span. Performance is not sensitive, however, to trial length beyond memory span length (Morris \& Jones, 1990; Postle et al., 2001). Thus, in each experiment presented in this report, we expected accuracy to be greater on 4-item than on 8- and 12-item trials, but to be comparable for the latter two. (Our previous experience indicated that reaction time $[\mathrm{RT}]$ is a less reliable index of many effects associated with this task; Postle et al., 2001.) Experiments 1 and 2 featured 32 trials of each length, occurring in a ran- domly determined order. Because 4-item trials served primarily as catch trials to ensure that participants would encode the first 4 items of each trial, Experiments 3 and 4 featured only sixteen 4 -item trials and 40 each of 8 - and 12-item trials. A testing session comprised training (verbal instructions followed by approximately 10 practice trials) and testing in four 24-trial blocks. All participants took a 5 -min break between the second and third blocks. The entire session lasted approximately $1 \mathrm{~h}$.

In Experiments 1, 2, and 3, group integrity was manipulated on all 8- and 12-item trials (i.e., on all trials that required updating). On half of all such trials, the final stimulus presentation event required the breakup of an encoding-def ined group. To illustrate, in the 8 -item trial featuring bt $d$ fr $x j p^{*} L^{*}$, the integrity of the $d f r$ group would be violated twice-when the presentation of $x j$ required dropping $d$ from the memory set, and when the presentation of $p$ required dropping $f$ from the memory set. It is this second instance of breaking up a group-because it was prompted by the final stimulus presentation event - that is of particular interest. This is because a previous study indicated that performance is insensitive to "midtrial" violations of group integrity (Postle et al., 2001). Thus, the grouping-related factor of principal interest in our experimental designs was whether or not the final stimulus presentation event of the trial prompted the violation of the integrity of a group currently in the memory set. An example of an 8-item trial that preserved group integrity would be $b \operatorname{tdf} r x j p * L^{*}$. Note, in this example, that although a violation of the $t d f$ group occurred with the presentation of $r x j$, this trial is classified as a group integrity-preserving trial because the onset of $p$, the final memorandum of the trial, did not result in a violation of the $r x j$ group. Indeed, in all the experiments that manipulated group integrity, violations of group integrity could occur unpredictably in portions of any trial preceding the final stimulus presentation epoch, including group integrity-preserving trials. In this way we ensured that violations of group integrity were not predictive of the end of the trial.

\section{EXPERIMENT 1}

The group integrity effect described by Postle et al. (2001) suggested that information about the context in which stimuli were presented was encoded in the mnemonic representation, in addition to information specific to stimulus identity. Although this effect was replicated and thus shown to be reliable, it remains unresolved whether it has broader implications for our understanding of STM. Its broader interest would be minimal, for example, if the group integrity effect arose from a strategy that participants adopted in response to the specific procedural characteristics of the updating task. Such a possibility is suggested in the paper that introduced the running memory span task to contemporary memory research. In it, Pollack and colleagues noted that, unprompted by them, participants "repeatedly reported that they attempted to group items in recall of materials" (Pollack, Johnson, \& Knaff, 1959, p. 142). And this and several subsequent studies have demonstrated that grouping strategies can improve STM performance (e.g., Hockey, 1973; Pollack et al., 1959; Ryan, 1969). Experiment 1 was designed as a first step toward addressing the question of strategy by assessing whether the encoding of contextual information is subject to volitional control. That is, if highly trained participants are told that attending to and encoding information about the context in 
which stimuli are presented (in this case, grouping) is deleterious to their performance, can they either block the encoding of this information or suppress its maintenance in STM? If they can, the effect of such instructions would be to abolish the group integrity effect. Such a demonstration would be inconsistent with the idea that the episodic code is fundamental to the representation of information in STM. The episodic coding model, in contrast, predicts that a group integrity effect would persist despite such explicit instructions to participants. Experiment 1 effected two tests of the volitional control of context effects in STM with two groups of participants, a "naive" group and an "instructed" group (naive and instructed with respect to the experimental factor of group integrity). In both cases, participants received thorough instruction about task procedure and were tested on a total of 192 trials. The naive group served as a control for the effects of experience and practice, independent of theoretically motivated instructions.

\section{Method}

Participants. We tested 70 participants, 35 in each of the two groups. We arrived at this $n$ by performing prospective power analysis with an effect size taken from Experiment 1B of Postle et al. (2001) and an estimate of variability taken from pilot data.

Procedure. Testing proceeded in two sessions of 96 trials each. The two sessions were separated by anywhere from $24 \mathrm{~h}$ to 10 days (pilot testing indicated that performance systematically declined in Session 2 when the two sessions were separated by only a 5-min break, perhaps due to fatigue and/or boredom). ISI was $3.5 \mathrm{sec}$. On 8 - and 12-item trials, valid probes matched memoranda with a lag of 3 or 4 items (i.e., in the first or second position in the memory set). This ensured that on group integrity-violating trials, the critical item was a member of the group that had been broken up just prior to the onset of the probe. On 4-item trials, valid probes matched items in all four positions in the memory set with equal probability. In Session 1, participants in the naive group were given standard instructions and performed 96 trials of the task, as described in the General Method section. Participants in the instructed group, in addition to the standard instructions, were also told about the nature of the group integrity factor in the experiment and that previous research had indicated that performance declines on group integrity-violating trials. Graphical illustrations of group integritypreserving and integrity-violating trials were used in this explanation. After confirming that participants in the instructed group understood the group integrity manipulation, we instructed them to seek to maximize their performance by deliberately ignoring or suppressing information about the grouping of items. This information, it was stressed, could not help performance, but could only hurt it. Prior to testing in Session 2, each participant received the same instructions that he/she had received prior to Session 1.

Design. Because the factor of group integrity, and the question of whether it would interact with group and/or session, was of principal theoretical interest in this experiment, accuracy and RT data were each analyzed with an omnibus analysis of variance (ANOVA) that included the factors of instructions (naive, instructed), group integrity (violated, preserved), probe validity (valid, invalid), and session $(1,2)$ to assess overall differences across the two groups. The rationale for including probe validity was that the knowledge of whether the group integrity effect is carried primarily by responses to valid or invalid probes may provide insight about the precise mechanisms underlying the effect. This analysis excluded 4-item trials, to which the factor of group integrity did not apply. The effect of trial length on accuracy (which included 4-item trials) was assessed in a second ANOVA incorporating the factors of instructions, trial length (4, 8, and 12 items), and session; the ANOVA was followed up with paired $t$ tests assessing the difference between accuracy at Length 4 versus the mean of Lengths 8 and 12-an index of the updating effect.

\section{Results}

Accuracy. The results of principal theoretical interest are presented in Table 1. The omnibus ANOVA revealed no effect of instructions $[F(1,68)=0.89$, n.s. $]$ or session $[F(1,68)=0.42$, n.s. $]$, but it did reveal main effects of group integrity $[F(1,68)=56.46, p<.0001]$ and probe validity $[F(1,68)=102.90, p<.0001]$; the only significant interaction was that of group integrity $X$ probe validity $[F(1,68)=41.81, p<.0001]$. Following up on this two-way interaction, a post hoc contrast that

Table 1

Mean Performance (\% Correct and RTs, With SEMs) by Instructions, Group Integrity, and Probe Validity, for Experiment 1

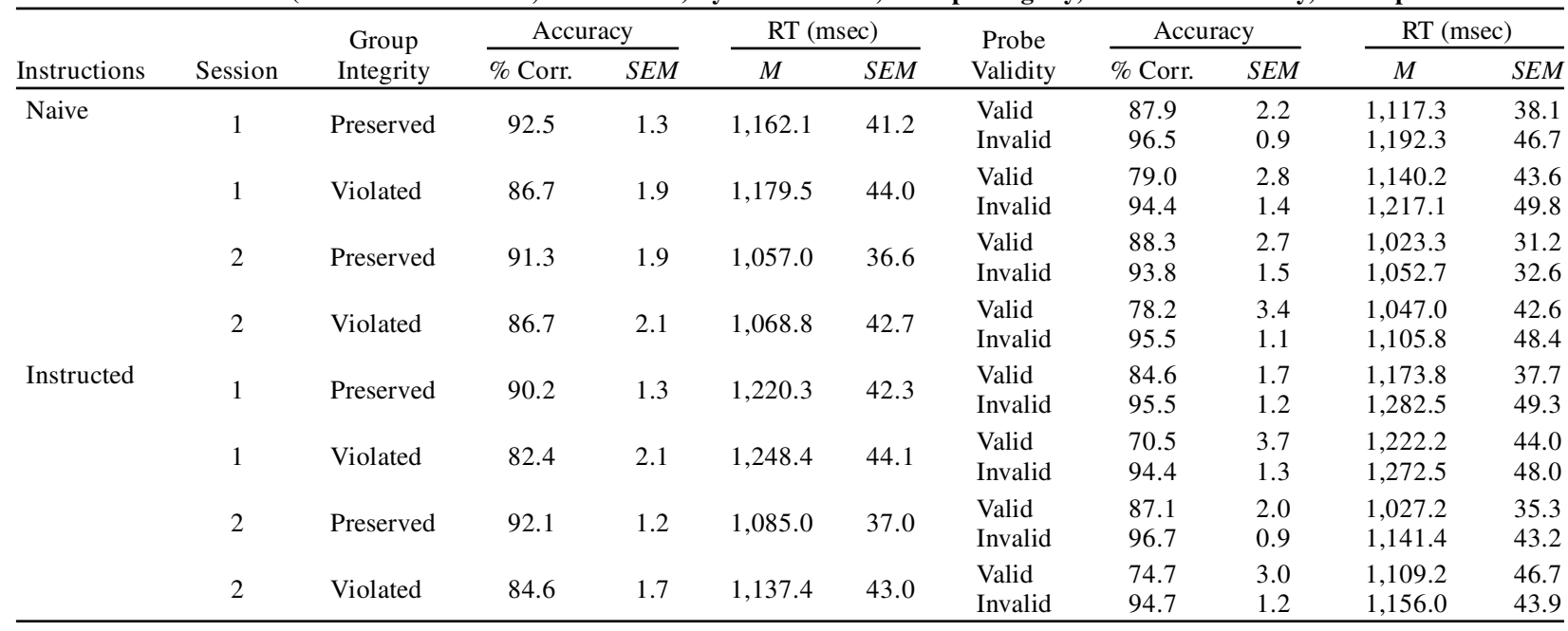

Note-The data in this table do not include 4-item trials. For performance on 4-item trials, see Table 2. 
collapsed across group and session confirmed that the group integrity effect was larger on valid than on invalid trials $[t(69)=6.50, p<.0001]$.

The analysis of accuracy as a function of trial length (Table 2) revealed no main effects of instructions $[F(1,68)=0.40$, n.s. $]$ or session $[F(1,68)=0.01$, n.s. $]$ but did reveal a main effect of length $[F(2,136)=10.09$, $p<.0001]$. The only significant interaction was that of instructions $\times$ trial length $[F(2,136)=3.43, p<.05]$, although the interaction of instructions $\times$ session approached significance $[F(2,136)=3 \cdot 22, p=.08]$. Planned contrasts of Length 4 versus the mean of Lengths 8 and 12 confirmed significant differences in three of the four group-by-session cells of this experiment-naive/ Session $1[t(34)=3.71, p<.001]$, instructed/Session 1 $[t(34)=3.68, p<.001]$, instructed/Session $2[t(34)=$ $2.43, p<.05]$ - and a marginally significant difference in naive/Session $2[t(33)=1.96, p=.058]$. (Data from 1 participant, whose Length 4 performance in Session 2 was at chance and was a statistical outlier, were excluded from this contrast.)

Reaction time. The omnibus ANOVA revealed no effect of instruction $[F(1,68)=1.25$, n.s.] but did reveal robust effects of session $[F(1,68)=63.33, p<.0001]$, group integrity $[F(1,68)=8.87, p<.005]$, and trial validity $[F(1,68)=43.16, p<.0001]$. The only significant interaction was that of instruction $\times$ group integrity $\times$ trial validity $[F(1,68)=6.04, p<.05]$. The $\mathrm{RT}$ data revealed a "fast-same" effect such as is commonly seen in yes/no recognition performance (Table 1; Proctor, 1986; Ratcliff, 1985; Sternberg, 1966).

\section{Discussion}

Performance of the naive and instructed groups mirrored each other, indicating that the detailed explanation of the group integrity manipulation to the latter group, coupled with the explicit instructions to ignore grouping and/or to suppress the influence of grouping, did not influence performance. Additionally, the effect did not di-

Table 2

Mean Performance by Trial Length, Experiment 1

\begin{tabular}{lcccccc}
\hline & \multirow{2}{*}{$\begin{array}{c}\text { Trial } \\
\text { Session }\end{array}$} & \multicolumn{2}{c}{ Accuracy } & & \multicolumn{2}{c}{ RT (msec) } \\
Nength & \% Corr. & SEM & & $M$ & SEM \\
\hline Naive Group & & & & & \\
1 & 4 & 92.7 & 1.5 & & $1,207.1$ & 42.5 \\
1 & 8 & 89.3 & 1.6 & & $1,180.2$ & 44.1 \\
1 & 12 & 89.7 & 1.6 & & $1,153.3$ & 41.1 \\
2 & 4 & 91.0 & 1.9 & & $1,088.4$ & 38.2 \\
2 & 8 & 87.6 & 2.1 & $1,074.6$ & 42.8 \\
2 & 12 & 90.9 & 1.9 & $1,055.5$ & 40.2 \\
Instructed Group & & & & & \\
1 & 4 & 91.5 & 1.0 & $1,287.9$ & 38.7 \\
1 & 8 & 86.6 & 1.4 & $1,234.0$ & 42.2 \\
1 & 12 & 85.8 & 2.0 & $1,249.6$ & 44.2 \\
2 & 4 & 91.9 & 1.2 & $1,150.3$ & 38.0 \\
2 & 8 & 89.5 & 1.3 & $1,110.8$ & 40.4 \\
2 & 12 & 86.9 & 1.9 & $1,106.3$ & 39.9 \\
\hline
\end{tabular}

minish with practice. That the group integrity effect was unaffected by instruction or by practice is consistent with the view that the factors underlying it-encoding and storage of the episodic code-cannot be controlled volitionally. Such inaccessibility to volitional control represents an initial piece of evidence for the idea that these cognitive operations are fundamental features of STM function.

Assessment of performance of both groups as a function of trial validity indicated that the group integrity effect was carried primarily by performance on valid trials. This difference may have been the product of a scaling artifact, because accuracy on invalid trials that preserved group integrity was near ceiling, and higher for both groups, in both sessions, than was accuracy on comparable valid trials. Experiment 2, which manipulated task difficulty, could offer an opportunity to assess this possibility. An alternative could be that violations of group integrity induced a conservative strategy in our participants: If these events had the effect of weakening mnemonic representations, the resultant decrease in confidence may have biased participants in favor of rejecting the probe.

\section{EXPERIMENT 2}

A possible objection to our interpretation of the results from Experiment 1 and from our previous studies (Postle et al., 2001) is that the relatively slow pace of the task (ISI of $3.5 \mathrm{sec}$ ) may have given participants more time than needed to execute the necessary encoding, discarding, and repositioning operations, as well as to consolidate the newly updated span of items. This may have left time to engage "optional" mental processes. Such an objection might derive from the set principle theory of Gorfein (1987), which posits that context effects in STM are a function of available encoding time. A variant of this view simply posits that previous versions of the task were too easy. We tested the plausibility of the time availability and the difficulty explanations of the results from Experiment 1 and from Postle et al. (2001) by decreasing the ISI, reasoning that reducing the time available to encode, discard, reposition, and consolidate would decrease the likelihood that participants would engage cognitive operations that were not necessary for task performance.

In line with the prediction of the difficulty hypothesis, there is evidence that grouping strategies can actually be harmful to STM performance at fast stimulus presentation rates. Hockey (1973) demonstrated that performance on an updating task declined as the presentation rate exceeded $1 \mathrm{~Hz}$ when participants were instructed to rehearse individually presented items in groups of three. In contrast, performance of participants instructed not to rehearse in groups improved with decreasing stimulus onset asynchrony (SOA; Hockey, 1973). So if the group integrity effect arises from an "input-processing strategy" that is under volitional control (as Hockey suggested), it is reasonable to assume that participants 
would abandon this strategy as its effect on performance reversed. We assessed this possibility in Experiment 2 by testing two groups of participants at presentation rates faster than that used in Experiment 1 and in our previous studies (Postle et al., 2001). In contrast to the time availability and difficulty explanations, the episodic coding theory predicted that the group integrity effect would be insensitive to changes in ISI. Independent of these two mutually incompatible predictions, we assumed that decreasing ISI would increase task difficulty, as indexed by accuracy.

\section{Method}

Participants. Sixteen participants were tested at an ISI of $2.5 \mathrm{sec}$, and 16 different participants were tested at an ISI of $2 \mathrm{sec}$.

Procedure. Apart from the ISIs, the updating task was identical to that used in Experiment 1. Subjective experience of the investigators, in addition to reports of pilot participants, indicated that participants cannot reliably sustain performance on our version of the updating task at ISIs shorter than $2 \mathrm{sec}-$ i.e., at SOAs shorter than $2.5 \mathrm{sec}$. At shorter ISIs, one simply cannot "keep up" with the stream of stimuli; one quickly loses the ability to update the memory set. Typically, participants resort to a strategy of simply monitoring the flow of stimuli and guessing about the probe stimulus by making a judgment about its familiarity. That is, they abandon attempts to discard and reposition items, and the task effectively ceases to be an updating task. Two seconds was thus the shortest ISI at which we could test participants and still be confident that they would be able to perform the updating task as we intended.

Design. As with Experiment 1, accuracy and RT data were each analyzed with omnibus 2 (ISI: $2.5,2.0) \times 2$ (group integrity) $\times 2$ (probe validity) ANOVAs designed to investigate group integrity effects. Because the effect of the ISI manipulation on the group integrity effect was of principal theoretical importance to this experiment, we planned pairwise analyses of this effect at each ISI if the factors of ISI and group integrity interacted in either the two- or three-way interaction. The effect of trial length on accuracy was assessed in a 2 (ISI) $\times 3$ (trial length) ANOVA followed up with paired $t$ tests assessing the difference between accuracy at Length 4 and the mean of Lengths 8 and 12 at each of the two ISIs.

\section{Results}

Accuracy. The ANOVA assessing group integrity effects in the accuracy data (Table 3 ) revealed no effect of ISI $[F(1,30)=0.18$, n.s.] but did reveal main effects of group integrity $[F(1,30)=70.05, p<.0001]$ and valid- ity $[F(1,30)=50.98, p<.0001]$. The only significant interaction was that of group integrity $\times$ validity $[F(1,30)=23.87, p<.0001]$.

Assessment of accuracy by trial length (Table 4) confirmed the updating effect: The ANOVA revealed no effect of ISI $[F(1,30)=2.13$, n.s.] but did reveal a significant effect of length $[F(2,60)=29.25, p<.0001]$; there was no interaction $[F(2,60)=0.79$, n.s.]. The planned contrasts confirmed the updating effect (Length 4 vs. the mean of Lengths 8 and 12) at both ISIs: $2.5 \mathrm{sec}[t(15)=$ $9.78, p<.0001]$ and $2 \sec [t(15)=4.93, p<.0005]$.

Reaction time. The omnibus ANOVA assessing group integrity effects in the RT data (Table 3 ) revealed no effects of ISI $[F(1,30)=0.02$, n.s.] or of validity $[F(1,30)=0.08$, n.s.] but did reveal a main effect of group integrity $[F(1,30)=11.51, p<.005]$. The group integrity $\times$ validity interaction was significant $[F(1,30)=$ $5.39, p<.05]$, and the three-way interaction approached significance $[F(1,30)=3.23, p=.08]$.

\section{Discussion}

Decreasing the ISI by $1 \mathrm{sec}$, to $2.5 \mathrm{sec}$, and by $1.5 \mathrm{sec}$, to $2 \mathrm{sec}$, had the effect of lowering overall performance (relative to the "standard" ISI of $3.5 \mathrm{sec}$ ), but the group integrity effect remained robust at these shorter ISIs. (Recall that the ISI of $2 \mathrm{sec}$ was the fastest paced task that we could administer and still be confident that participants were following instructions.) These results therefore permit us to reject the difficulty explanation of the group integrity effect. They also challenge the encoding time limitation aspect of the set principle theory (Gorfein, 1987). The results of Experiment 2 are consistent, however, with our contention that the episodic code is fundamental to STM representations.

As was the case with Experiment 1, the group integrity effect was much more pronounced on valid than on invalid trials. This was even true on the most difficult ( 2 sec ISI) trials, although for these trials the group integrity effect seemed to be larger for invalid trials than it had been on the longer ISI trials of Experiments 1 and 2. Conclusive unconfounding of scaling effects from other factors that may differentiate group integrity effects on

Table 3

Mean Performance (\% Correct and RTs, with SEMs) by Group Integrity and Probe Validity, for Experiment 2

\begin{tabular}{|c|c|c|c|c|c|c|c|c|c|c|}
\hline \multirow[b]{2}{*}{ ISI } & \multirow{2}{*}{$\begin{array}{l}\text { Group } \\
\text { Integrity }\end{array}$} & \multicolumn{2}{|c|}{ Accuracy } & \multicolumn{2}{|c|}{ RT (msec) } & \multirow{2}{*}{$\begin{array}{c}\text { Probe } \\
\text { Validity }\end{array}$} & \multicolumn{2}{|c|}{ Accuracy } & \multicolumn{2}{|c|}{ RT (msec) } \\
\hline & & $\%$ Corr. & SEM & $M$ & $S E M$ & & $\%$ Corr. & $S E M$ & $M$ & $S E M$ \\
\hline \multirow[t]{4}{*}{$2.5 \mathrm{sec}$} & \multirow{2}{*}{ Preserved } & \multirow{2}{*}{91.9} & \multirow{2}{*}{1.4} & \multirow{2}{*}{$1,339.4$} & \multirow{2}{*}{60.8} & Valid & 86.2 & 2.4 & $1,296.6$ & 49.5 \\
\hline & & & & & & Invalid & 96.0 & 1.5 & $1,382.2$ & 75.1 \\
\hline & \multirow[t]{2}{*}{ Violated } & \multirow[t]{2}{*}{79.9} & \multirow[t]{2}{*}{2.1} & \multirow[t]{2}{*}{$1,417.3$} & \multirow[t]{2}{*}{63.9} & Valid & 64.3 & 4.4 & $1,458.9$ & 73.1 \\
\hline & & & & & & Invalid & 93.9 & 1.5 & $1,375.8$ & 59.1 \\
\hline \multirow{3}{*}{$2.0 \mathrm{sec}$} & Preserved & 90.1 & 1.7 & $1,343.8$ & 63.7 & Valid & 86.7 & 2.9 & $1,347.1$ & $\begin{array}{l}57.6 \\
81.9\end{array}$ \\
\hline & \multirow{2}{*}{ Violated } & \multirow{2}{*}{76.4} & \multirow{2}{*}{3.0} & \multirow{2}{*}{$1,389.1$} & \multirow{2}{*}{68.0} & Valid & 66.5 & 4.9 & $1,403.1$ & 69.3 \\
\hline & & & & & & Invalid & 88.5 & 2.6 & $1,375.1$ & 78.3 \\
\hline
\end{tabular}

Note-The data in this table do not include 4-item trials. For performance on 4-item trials, see Table 4. ISI, interstimulus interval. 
Table 4

Mean Performance (\% Correct and RTs, With SEMs) by Trial Length, for Experiment 2

\begin{tabular}{lcccccc}
\hline & \multirow{2}{*}{$\begin{array}{c}\text { Trial } \\
\text { ISI }\end{array}$} & \multicolumn{2}{c}{ Accuracy } & & \multicolumn{2}{c}{ RT (msec) } \\
\cline { 3 - 4 } \cline { 6 - 7 } $2.5 \mathrm{sec}$ & 4 & 95.7 & 1.1 & & $1,429.4$ & 243.2 \\
& 8 & 84.7 & 2.0 & & $1,409.8$ & 195.5 \\
& 12 & 86.9 & 1.7 & & $1,394.5$ & 218.9 \\
$2.0 \mathrm{sec}$ & 4 & 90.8 & 3.4 & & $1,437.0$ & 237.3 \\
& 8 & 82.9 & 3.5 & & $1,413.1$ & 246.6 \\
& 12 & 82.3 & 3.1 & & $1,442.7$ & 249.0 \\
\hline
\end{tabular}

Note-ISI, interstimulus interval.

valid versus invalid trials will require an experimental design that manipulates difficulty by means other than the ISI.

\section{EXPERIMENT 3}

Experiments 1 and 2 established the robustness of the group integrity effect in the updating task when it is administered in a yes/no recognition format. The principal goal of Experiment 3 was to determine whether or not this effect generalizes to a procedure other than that used in the previous two experiments (and in Postle et al., 2001). We did this by changing the response required in the updating task from yes/no recognition of a memory probe to immediate serial recall. Additionally, we designed this experiment to explore questions about the mechanisms underlying the group integrity effect. To this point we have simply used the group integrity effect as an index of the episodic code in STM. In this experiment, we also tested the memory set-size hypothesis, which might explain how the episodic code influences performance via the group integrity effect. According to this hypothesis, the mnemonic representations of items that are encoded as a group may be associated more strongly (Raaijmakers \& Shiffrin, 1981; Wickelgren, 1968) than items that are adjacent in the memory set, but that were not encoded together. ${ }^{2}$ As a result, the probability of successful discarding of an item from the memory set might be lower on trial epochs requiring a violation of group integrity than on those that do not. By this hypothesis, the group integrity effect amounts to a memory set-size (or "load") effect, in which, on average, a larger number of items are held in STM on group integrityviolating than on group integrity-preserving trials. (A similar mechanism has been proposed by Bunge, Ochsner, Desmond, Glover, \& Gabrieli, 2001, to explain proactive interference effects in working memory.) Independent of the memory set-size hypothesis, the design of Experiment 3 would also permit us to investigate performance by serial position, a measure that might also provide insight about the nature of the group integrity effect.

\section{Method}

Participants. Fifteen individuals participated in this experiment.

Procedure. Trials differed from those of Experiment 1 only in that the probe on each trial featured a question mark bounded by as- terisks $(*$ ? *), which prompted participants to write the memory span, in the correct order, on a response sheet. They were instructed to always give four responses on each trial, and omissions were scored as errors. Of particular interest were trials featuring "intrusion" errors, in which an item with a lag of 5 or 6 was incorrectly recalled as having been in the final memory span. On group integritypreserving trials, these critical items belonged to the group that was discarded during the final stimulus presentation epoch; on group integrity-violatin $g$ trials, they belonged to the group whose integrity was violated on the final stimulus presentation epoch. (On six group integrity-preserved trials, the critical item had a lag of 7, because it was the first in a group of three.) Similar methods have been used successfully to assess the factors underlying positional coding in STM (Henson, 1999). The memory set-size hypothesis predicted a greater proportion of intrusion errors on group integrityviolating than integrity-preserving trials, because of the postulated higher probability that lag 5 and lag 6 items would still be maintained in the memory set.

The response sheet was an $8.5 \times 11$ in. piece of paper on which there appeared 24 rows with four underscores ("__") per row. Each response sheet corresponded to the number of trials in a block, and, thus, four were used per experiment. Data from each participant were scored by hand, independently, by two scorers. The results obtained by each scorer were then compared with each other as a means of double checking these results, and trials receiving inconsistent scores were rechecked to resolve the discrepancy.

Design. The critical test of the memory set-size hypothesis was the pairwise contrast between the proportion of error trials of each trial type (group integrity preserved vs. violated) that were intrusion errors. Serial position effects were assessed by calculating performance by position and submitting these data to a 3 (trial type: group integrity preserved, group integrity violated, 4 -item) $\times 4$ (position) ANOVA. Because the numbers of trials per length were not proportional in these experiments (see Procedure section under General Method), overall performance by trial length was not assessed.

\section{Results}

Overall performance ( $71.9 \%$ correct) was markedly lower than it had been on all previous experiments (each of which had featured a "yes"/"no" recognition procedure). The contrast assessing the difference in performance between group integrity-preserved (mean percentage correct $=78.2, S E M=2.5$ ) and group integrity-violated (mean percentage correct $=60.0, S E M=4.0$ ) trials confirmed a robust group integrity effect $[t(14)=7.70, p=$ $.0001]$. In dramatic contrast to the prediction of the memory set-size hypothesis, however, we found that the proportion of intrusion errors on group integrity-preserving trials $(M=0.453, S E M=0.054)$ was significantly greater than the proportion of intrusion errors on group integrityviolating trials $(M=0.287, S E M=0.031)[t(14)=3.27$, $p<.01]$.

Performance by serial position, illustrated in Figure 1, was disproportionately poor at longer lags on group integrity-violating trials. The $3 \times 4$ ANOVA revealed main effects of trial type $[F(2,28)=21.79, p<.0001]$ and position $[F(3,42)=6.73, p<0.001]$, as well as an interaction $[F(6,84)=4.80, p<.0005]$.

\section{Discussion}

The results of Experiment 3 revealed a robust group integrity effect in an updating task that required immediate serial recall at the end of each trial, thereby demon- 


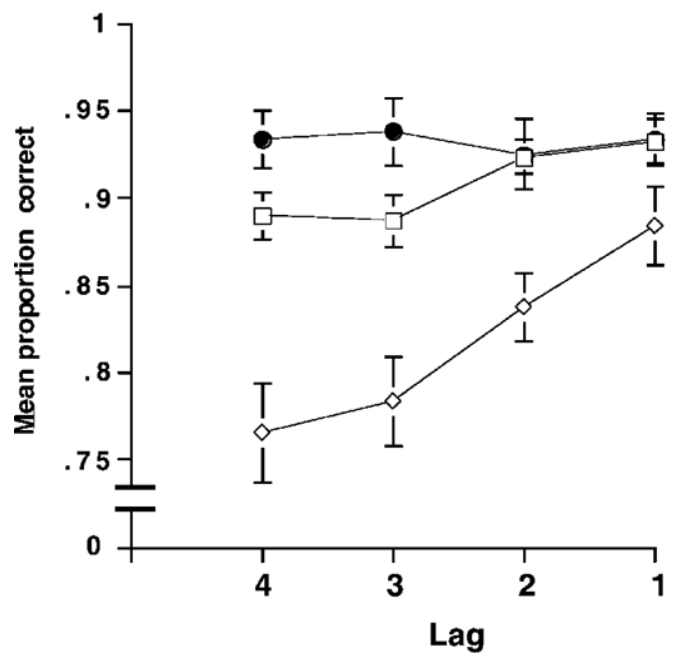

Trial type

$\square-$ Group integrity preserved

$\curvearrowright$ Group integrity violated

4-item

Figure 1. Results of Experiment 3, plotted as a function of trial type and of serial position within the memory set. ("Lag" refers to ordinal lag of a memorandum's presentation with respect to the probe; error bars represent $S E M$ s.)

strating that this effect generalizes across response procedures. They also revealed a higher rate of intrusion errors associated with group integrity-preserving than integrity-violating trials, despite the significantly higher number of errors on the latter type of trials. These results thus also represent a convincing rejection of the memory set-size hypothesis of the group integrity effect. Finally, the serial position data, indicating that performance on group integrity-violating trials was worst at longer lags from the probe, suggest that violations of group integrity may have their most pronounced effects by weakening the representation of items belonging to the group that was broken up.

On the basis of these results, we proffer a new hypothesis about the nature of this effect: Stimulus presentation epochs requiring a violation of group integrity may load additional stress on the discarding operation, and this stress can disrupt the contents of the memory set. Stated more generally, engaging control operations that conflict with the episodic code may result in a weakening of stimulus representations in STM. An alternative hypothesis comes from Johnson (1972), who proposed a "hierarchical coding" model in which individual items are chunked during the encoding process, and each chunk is represented by a single code. These "lower level" codes could themselves be chunked and represented by a "higher order" code. In such a scheme, recoding of individual memoranda would be problematic: "For example, if SBJFQLZ is chunked as SB JFQ LZ at the lowest level, with a single code representing each chunk, it would not be possible to recode the sequence at the next level into a code for SBJ and another for FQLZ because the higher order code that represented the code for JFQ would also have to represent the codes for J, F, and Q" (Johnson, 1972, p. 138). That is, upon decoding, J would be represented twice. Empirical work (Johnson, 1969,
1970, 1972) has suggested that experimental manipulations requiring such recoding operations result in dramatic loss of memory for all items in STM. It was as though, Johnson (1972) analogized, the original lower level codes acted as opaque containers whose contents could not be discerned by inspection from the outside, and whose breaking would result in loss of the contents in the container. When the results of the present experiment are viewed from this perspective, they suggest that group integrity violations may require decoding operations that entail breaking the "opaque containers" that represent groups of letters, along with a resultant loss of the elements making up the group. Additional work will be required to assess these two models of the group integrity effect.

\section{EXPERIMENT 4}

All of the data presented thus far emphasize the effect of varying one experimental factor-group integrity. But do context effects in STM generalize to other kinds of information? The answer to this question has important implications for the episodic coding theory. Experiment 4 addressed this question by investigating whether contextual information about color, a domain of information that is unrelated to temporal grouping and that cannot be related to order, would also influence performance on an updating task. Additionally, the results of Experiment 4 would be important for the validation of our interpretation of the results of the previous three experiments. This is because the group integrity-related results produced by these experiments are vulnerable to at least two alternative interpretations. According to one alternative explanation, the effects that we have reported may be specific to this particular combination of procedures and materials. That is, because successful perfor- 
mance of the updating task depends on encoding and maintaining positional information with high fidelity, the updating task may induce participants to encode all information that might possibly be related to item order, including grouping. From this perspective, the group integrity effect may just be an empirical by-product of our method and of minimal broader theoretical interest. A second alternative explanation of the results of the first three experiments is that what we are calling the "group integrity effect" may have nothing at all to do with the manipulation of context. According to this second alternative explanation, group integrity-preserving trials may, on average, present a more "regular" or predictable progression of groups, whereas the grouping of items on group integrity-violating trials is more "irregular" or unpredictable. This difference, which would have nothing to do with group integrity, could result in a disparity of difficulty on the two types of trials. Experiment 4, which also assesses the existence of context effects in STM, is not vulnerable to either of these alternative explanations. Evidence of color context effects in STM, therefore, would bolster our confidence in our interpretations of the results of Experiments 1-3.

\section{Method}

Participants. We tested 35 participants in this experiment. Preliminary testing confirmed that all could identify and distinguish among the four colors employed in this experiment with no difficulty.

Procedure. Memoranda were presented individually, with an ISI of $2.5 \mathrm{sec}$. (The selection of this ISI was arbitrary and inconsequential to the theoretical interpretation of the results of this experiment, because Experiments 1 and 2 had produced robust context effects across the range of ISIs from 3.5 down to $2 \mathrm{sec}$.) Each stimulus was presented in one of four colors - red, yellow, green, or blue-against a black background. Colors of memoranda were determined pseudorandomly, with the only constraint being that each color appeared an equal number of times during each block of 24 trials. Probe stimuli were also presented in one of these four colors. Each invalid probe matched a lure that had appeared among the first 4 items on 8 -item trials and among the first 8 items on 12 -item trials. The color of the probe matched the color of its associated match or lure with a probability of .5 . On any trial type, the color of the probe could match that of one or several noncritical memoranda (this was not controlled). The color feature of the stimuli was described to participants prior to training, and they were told that color information was unrelated to the memory demands of the task.

Design. The factor of color congruity between the probe and the critical memorandum was orthogonal to that of trial validity. Color congruity was controlled in such a way that color matched between the probe and the critical memorandum on one half of the trials of each trial length. The episodic coding model predicted an effect of color congruity on performance. More specif ically, we predicted that the color congruity manipulation would induce participants to false alarm to lures that matched the color of the probe (i.e., performance would be lower on invalid/color-congruent trials). Note that this is the opposite of the pattern seen in the group integrity effect, which is manifested primarily as incorrect rejections of valid probes. In terms of our experimental design, we predicted that a $2 \times 2$ ANOVA with the factors of color congruity (congruent, incongruent) and probe validity (valid, invalid) would reveal an interaction of these two factors. Were there to be an effect of color congruity on responses to valid probes, it would be expected to be one of facilitation (i.e., performance would be higher on valid/colorcongruent trials, producing a crossover interaction).
Because the numbers of trials per length were not proportional in these experiments (see Procedure section under General Method), overall performance by trial length was not assessed.

\section{Results}

Overall performance was $88.6 \%$ correct. The $2 \times 2$ ANOVA revealed main effects of color congruity $[F(1,34)$ $=4.35, p<.05]$ and trial validity $[F(1,34)=17.64, p<$ $.005]$ and an interaction $[F(1,34)=5.01, p<.05]$. Pairwise comparisons confirmed that color congruity affected performance (mean percentage correct $[S E M]$ ) on invalid trials [incongruent/invalid 92.9 (1.1); congruent/invalid $88.4(1.7) ; t(34)=3.0, p<.005$ ] but not on valid trials [incongruent/valid 82.5 (1.7); congruent/ valid $83.2(1.6) ; t(34)=0.5$, n.s.; Figure 2$]$.

\section{Discussion}

Controlling the color of stimuli had an effect on updating performance: a color congruity effect. This result demonstrates that context effects in STM generalize beyond factors that relate to grouping. This is theoretically important because it suggests that episodic codes in STM can incorporate information that is unequivocally unrelated to the demands of the task. Thus, the encoding and maintenance in STM of episodic information may not be limited to factors that relate directly to performance strategy or task demands, but may apply to any type of contextual information that is intrinsic to stimuli.

The color congruity effect is similar to the group integrity effect in that each indexes the effect of context in STM. But the two differ in terms of the stage of processing that each affects. The factors underlying the color congruity effect cannot come into play until the congruity of color between the probe and the critical memorandum on the trial can be apprehended. This effect, therefore, derives from the decision and/or re-

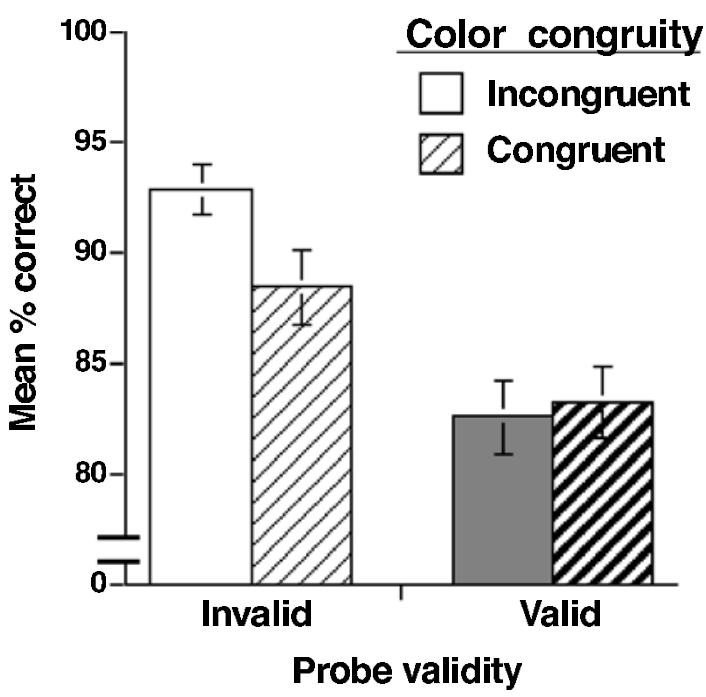

Figure 2. Results of Experiment 4, illustrating that the color congruity effect manifests itself on invalid trials. (Error bars represent $S E M$ s.) 
sponse processes that are prompted by the onset of the probe. The group integrity effect, in contrast, is attributable to the presentation of a memorandum that prompts a violation of group integrity. It derives, therefore, from the control processes involved in the updating of the memory set, perhaps the putative discarding and repositioning operations considered in the introduction.

\section{GENERAL DISCUSSION}

The results from these experiments affirm the episodic coding hypothesis: They suggest that the episodic code in STM is not subject to volitional control; that it is not diminished as task difficulty increases; that it generalizes across response procedure; and that it generalizes across varieties of contextual information, including information that is unequivocally unrelated to task demands. These results are therefore consistent with the hypothesis that an episodic code is fundamental to representations in STM. This represents an extension of previous work in this area by Wickens, Gorfein, and others (e.g., Gorfein \& Hoffman, 1987; Wickens, 1973) and reemphasizes the complexity of encoding operations and of stimulus representation in STM. It also suggests that context alpha is an important factor for which successful formal models of STM must account.

Because all of the experiments presented in this report employed tests of recognition or recall from a running span of letters (i.e., updating), their results leave unresolved at least three important issues about the nature of context effects in STM: (1) whether they generalize to other STM tasks, (2) whether they generalize to STM for other domains of information (e.g., visuospatial or tactile), and (3) whether they generalize to extrinsic (e.g., environmental or intrinsically generated) context. With respect to the first point, most theorists would agree that the updating task places demands on working memory, because of its requirements for executive control. And because there is evidence for a functional distinction between working memory and STM (e.g., Cowan, 1995; D'Esposito \& Postle, 2000; Engle, Tuholski, Laughlin, \& Conway, 1999), it will be important to demonstrate that tests that are unequivocal measures of STM, such as item recognition, are also sensitive to manipulations of context. With respect to the third point, our manipulations of context alpha were limited to intrinsic (or intra-item) contexts (Bjork \& Richardson-Klavehn, 1989). Thus, the effects on STM of manipulating environmental context (e.g., room cues, Bjork \& Richardson-Klavehn, 1989, or aquatic submersion, Godden \& Baddeley, 1975) and internally generated context (such as temporal distinctiveness, Glenberg \& Swanson, 1986, or emotional or physical sensations, Raaijmakers \& Shiffrin, 1981) remain to be explored.

With regard to the group integrity effect, the data generated by Experiment 3 rule out a memory set-size explanation for this effect. Two plausible alternatives that await empirical validation are that engaging control operations that conflict with the episodic code may result in a weakening of stimulus representations in STM, or that violations of group integrity engage a recoding process that results in a loss of information from STM (Johnson, 1972).

\section{REFERENCES}

Baddeley, A. D. (1982). Domains of recollection. Psychological Review, 89, 708-729.

Baddeley, A. D., \& Logie, R. H. (1999). Working memory: The multiple-component model. In A. Miyake \& P. Shah (Eds.), Models of working memory (pp. 28-61). Cambridge: Cambridge University Press.

BJork, R. A., \& Richardson-KLAVEHn, A. (1989). On the puzzling relationship between environmental context and human memory. In C. Izawa (Ed.), Current issues in cognitive processes: The Tulane Flowerree Symposium on Cognition (pp. 313-344). Hillsdale, NJ: Erlbaum.

Bunge, S. A., Ochsner, K. N., Desmond, J. E., Glover, G. H., \& GABRIELI, J. D. E. (2001). Prefrontal regions involved in keeping information in and out of mind. Brain, 124, 2074-2086.

Cowan, N. (1988). Evolving conceptions of memory storage, selective attention, and their mutual constraints within the human information processing system. Psychological Bulletin, 104, 163-171.

Cowan, N. (1995). Attention and memory: An integrated framework. New York: Oxford University Press.

CoWAn, N. (1999). An embedded-processes model of working memory. In A. Miyake \& P. Shah (Eds.), Models of working memory (pp. 62-101). Cambridge: Cambridge University Press.

CowAn, N. (2001). The magical number 4 in short-term memory: A reconsideration of mental storage capacity. Behavioral \& Brain Sciences, 24, 87-185.

D'Esposito, M., \& Postle, B. R. (1999). The dependence of span and delayed-response performance on prefrontal cortex. Neuropsychologia, 37, 1303-1315.

D'Esposito, M., \& Post Le, B. R. (2000). Neural correlates of processes contributing to working memory function: Evidence from neuropsychological and pharmacological studies. In S. Monsell \& J. Driver (Eds.), Control of cognitive processes: Attention and performance XVIII (pp. 579-602). Cambridge, MA: MIT Press.

Engle, R. W., Kane, M. J., \& Tuholski, S. W. (1999). Individual differences in working memory capacity and what they tell us about controlled attention, general fluid intelligence, and functions of the prefrontal cortex. In A. Miyake \& P. Shah (Eds.), Models of working memory (pp. 102-134). Cambridge: Cambridge University Press.

Engle, R. W., Tuholski, S. W., Laughlin, J. E., \& Conway, A. R. A. (1999). Working memory, short-term memory, and general fluid intelligence: A latent-variable approach. Journal of Experimental Psychology: General, 128, 309-331.

Glenberg, A. M., \& Swanson, N. G. (1986). A temporal distinctiveness theory of recency and modality effects. Journal of Experimental Psychology: Learning, Memory, \& Cognition, 12, 3-15.

Godden, D. R., \& BADdeley, A. D. (1975). Context-dependent memory in two natural environments: On land and under water. British Journal of Psychology, 66, 325-331.

GorfeIN, D. S. (1987). Explaining context effects on short-term memory. In D. S. Gorfein \& R. R. Hoffman (Eds.), Learning and memory: The Ebbinghaus Centennial Conference (pp. 153-172). Hillsdale, NJ: Erlbaum.

GorfeIN, D. S., \& HofFMAN, R. R. (EDS.) (1987). Learning andmemory: The Ebbinghaus Centennial Conference. Hillsdale, NJ: Erlbaum.

HASHER, L., \& ZACKS, R. T. (1988). Working memory, comprehension, and aging: A review and a new view. In G. H. Bower (Ed.), The psychology of learning and motivation (Vol. 22, pp. 193-225). San Diego: Academic Press.

Henson, R. N. A. (1999). Positional information in short-term memory: Relative or absolute? Memory \& Cognition, 27, 915-927.

Hockey, R. (1973). Rate of presentation in running memory and direct 
manipulation of input-processing strategies. Quarterly Journal of Experimental Psychology, 25, 104-111.

Johnson, N. F. (1969). Chunking: Associative chaining versus coding. Journal of Verbal Learning \& Verbal Behavior, 8, 725-731.

JoHnson, N. F. (1970). Chunking and organization in the process of recall. In G. H. Bower (Ed.), The psychology of learning and motivation (Vol. 4, pp. 171-247). New York: Academic Press.

JoHnSON, N. F. (1972). Organization and the concept of a memory code. In A. W. Melton \& E. Martin (Eds.), Coding processes in human memory (pp. 125-160). Washington, DC: Winston.

Jonides, J., Smith, E. E., Marshuetz, C., Koeppe, R. A., \& ReuterLORENZ, P. A. (1998). Inhibition in verbal working memory revealed by brain activation. Proceedings of the National Academy of Sciences, 95, 8410-8413.

Kieras, D. E., Meyer, D. E., Mueller, S., \& Seymour, T. (1999). Insights into working memory from the perspective of the EPIC architecture for modeling skilled perceptual-motor and cognitive human performance. In A. Miyake \& P. Shah (Eds.), Models of working memory (pp. 183-223). Cambridge: Cambridge University Press.

Kiss, I., Pisio, C., Francois, A., \& Schopflocher, D. (1998). Central executive function in working memory: Event-related brain potential studies. Cognitive Brain Research, 6, 235-247.

LogIE, R. H. (1995). Visuo-spatial working memory. Hove, U.K.: Erlbaum.

Lovett, M. C., Reder, L. M., \& Lebiere, C. (1999). Modeling working memory in a unified architecture: An ACT-R perspective. In A. Miyake \& P. Shah (Eds.), Models of working memory (pp. 135-182). Cambridge: Cambridge University Press.

McElree, B. (2001). Working memory and focal attention. Journal of Experimental Psychology: Learning, Memory, \& Cognition, 27, 817835.

Morris, N., \& Jones, D. M. (1990). Memory updating in working memory: The role of the central executive. British Journal of Psychology, 81, 111-121.

NAIRne, J. S. (2002). Remembering over the short-term: The case against the standard model. Annual Review of Psychology, 53, 53-81.

O'Reilly, R. C., Braver, T. S., \& Cohen, J. D. (1999). A biologically based computational model of working memory. In A. Miyake \& P. Shah (Eds.), Models of working memory (pp. 375-411). Cambridge: Cambridge University Press.

Pollack, I., Johnson, L. B., \& Knaff, P. R. (1959). Running memory span. Journal of Experimental Psychology, 57, 137-146.

Postle, B. R., Berger, J. S., Goldstein, J. H., Curtis, C. E., \& D'Esposito, M. (2001). Behavioral and neurophysiological correlates of episodic coding, proactive interference, and list length effects in a running span verbal working memory task. Cognitive, Affective, \& Behavioral Neuroscience, 1, 10-21.

Proctor, R. W. (1986). Response bias, criteria settings, and the fastsame phenomenon: A reply to Ratcliff. Psychological Review, 93, 473-477.

RaAijmakers, J. G. W., \& Shiffrin, R. M. (1981). Search of associative memory. Psychological Review, 88, 93-134.
RATCLIFF, R. (1985). Theoretical interpretations of the speed and accuracy of positive and negative responses. Psychological Review, 92, 212-225.

RyAN, J. (1969). Grouping and short-term memory: Different means and patterns of grouping. Quarterly Journal of Experimental Psychology, 21, 137-147.

Salmon, E., Van der Linden, M., Collette, F., Delfiore, G., MAQuet, P., Degueldre, C., Luxen, A., \& Franck, G. (1996). Regional brain activity during working memory tasks. Brain, 119, $1617-$ 1625.

Smith, S. M., \& Vela, E. (2001). Environmental context-dependent memory: A review and meta-analysis. Psychonomic Bulletin \& Review, 8, 203-220.

Sternberg, S. (1966). High-speed scanning in human memory. Science, 153, 652-654.

Stuss, D. T., \& KNight, R. T. (EDs.) (2002). Principles of frontal lobe function. New York: Oxford University Press.

WiCKelgren, W. A. (1968). Sparing of short-term memory in an amnesic patient: Implications for strength theory of memory. Neuropsychologia, 6, 235-244.

WiCKens, D. D. (1973). Some characteristics of word encoding. Memory \& Cognition, 1, 485-490.

WiCKENS, D. D. (1987). The dual meanings of context: Implications for research, theory, and applications. In D. S. Gorfein \& R. R. Hoffman (Eds.), Learning and memory: The Ebbinghaus Centennial Conference (pp. 135-152). Hillsdale, NJ: Erlbaum.

\section{NOTES}

1. In this report we are purposefully noncommittal about the theoretical organization of STM because we believe that the episodic coding hypothesis has implications for many models of the retention of information over short periods of time, including "working memory" of the multiple-component model (Baddeley \& Logie, 1999; Logie, 1995), the EPIC architecture (Kieras, Meyer, Mueller, \& Seymour, 1999), the biologically based computational model of O'Reilly and colleagues (O'Reilly, Braver, \& Cohen, 1999), and most neuroscientifically motivated models (e.g., Stuss \& Knight, 2002), as well as the view of STM as the activation of subsets of long-term memory representations as it is variously implemented by, for example, the embedded-processes model (Cowan, 1988, 1999), the controlled-attention model (Engle, Kane, \& Tuholski, 1999), and the ACT-R cognitive architecture (Lovett, Reder, \& Lebiere, 1999). Whether it also has implications for models that deny a fundamental distinction between STM and long-term memory (as reviewed by Nairne, 2002), however, is unclear.

2. This idea arose during discussions with Rik Henson.

(Manuscript received April 3, 2002;) revision accepted for publication June 20, 2003.) 